\title{
Mosaicking and enhancement of slit lamp biomicroscopic fundus images
}

Sarnoff Corporation, Princeton, NJ, USA

$\mathrm{J}$ Asmuth

P Sajda

Computer Vision Laboratory, Scheie Eye Institute, University of Pennsylvania,

Philadelphia, PA, USA

B Madjarov

J W Berger

Correspondence to:

Jeffrey W Berger, MD, PhD, Computer Vision

Laboratory, Scheie Eye

Institute, University of

Pennsylvania, Philadelphia,

PA 19104, USA

jwberger@

mail.med.upenn.edu

Accepted for publication 14 December 2000

Jane Asmuth, Bojidar Madjarov, Paul Sajda, Jeffrey W Berger

\begin{abstract}
Aims-To process video slit lamp biomicroscopic fundus image sequences in order to generate wide field, high quality fundus image montages which might be suitable for photodocumentation.

Methods-Slit lamp biomicroscopic fundus examination was performed on human volunteers with a contact or non-contact lens. A stock, charge coupled device camera permitted image capture and storage of the image sequence at 30 frames per second. Acquisition time was approximately 30 seconds. Individual slit lamp biomicroscope fundus image frames were aligned and blended with custom developed software.
\end{abstract}

Results-The developed algorithms allowed for highly accurate alignment and blending of partially overlapping slit lamp biomicroscopic fundus images to generate a seamless, high quality, wide field montage.

Conclusions-Video image acquisition and processing algorithms allow for mosaicking and enhancement of slit lamp biomicroscopic fundus images. The improved quality and wide field of view may confer suitability for inexpensive, real time photodocumentation of disc and macular abnormalities.

(Br f Ophthalmol 2001;85:563-565)

Fundus cameras provide wide field, high quality images of posterior segment structures including the optic disc and macula. However, the slit lamp biomicroscope, the workhorse for ophthalmic diagnosis and treatment, is far more ubiquitous, and is now often equipped with video attachments to permit image capture for documentation, storage, and transmission. In many cases, image quality may be low, in part attributable to a narrow field of
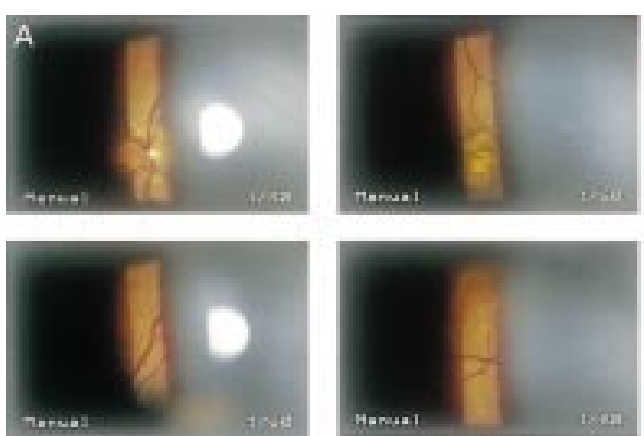

Figure 1 (A) Unprocessed slit lamp biomicroscopic fundus images of an eye with presumed ocular histoplasmosis syndrome following laser treatment for juxtafoveal choroidal neovascularisation. (B) Slit lamp fundus biomicroscopic

images following automated extraction of the illuminated portion of the fundus and elimination of specular reflections. view and specular reflections arising from the cornea, sclera, and hand held lens.

In this journal, we have previously demonstrated successful, real time extraction of the illuminated portion of fundus from slit lamp biomicroscope fundus image video sequences. ${ }^{1}$ In the present study, we sought to process video slit lamp biomicroscopic fundus image sequences in order to generate wide field, high quality fundus image mosaics which might be more suitable for photodocumentation of fundus features and discernment of change based on images acquired over time.

\section{Methods}

With appropriate institutional review board approval, and following informed consent, slit lamp (Nikon NS-1V) biomicroscopic fundus examination was performed on human volunteers with a contact lens or 78 dioptre hand held non-contact lens. A stock, charge coupled device (CCD) camera attached to one of the oculars through a beam splitter permitted image capture and storage of the image sequence at 30 frames per second to S-video tape.

Fundus image sequences, derived during video slit lamp biomicroscopic examination, are of lower quality and contrast, and with specular reflections degrading the true signal with noise (Fig 1A). That portion of the image corresponding to the illuminated portion of the fundus may be extracted automatically from the incoming video image sequence based on colour content and shape constraints (Fig 1B). While the fundus image has little blue colour content, specular reflections from the cornea, sclera, and contact lens were noted to have significant brightness contributions in the blue channel. The illuminated portion of the fundus in the slit lamp biomicroscope image corresponds to the single, contiguous, vertically elongated blob of pixels with brightness in
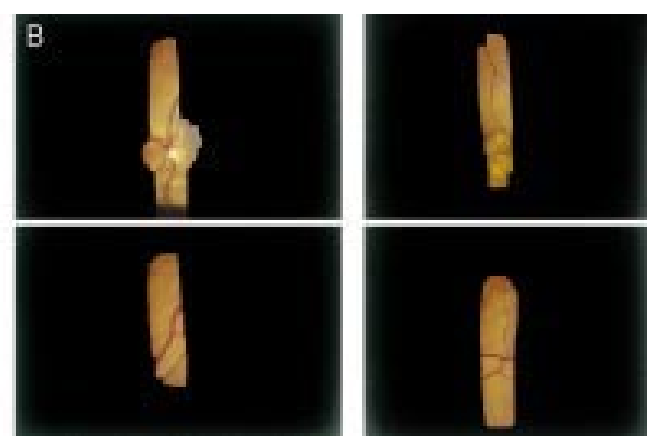

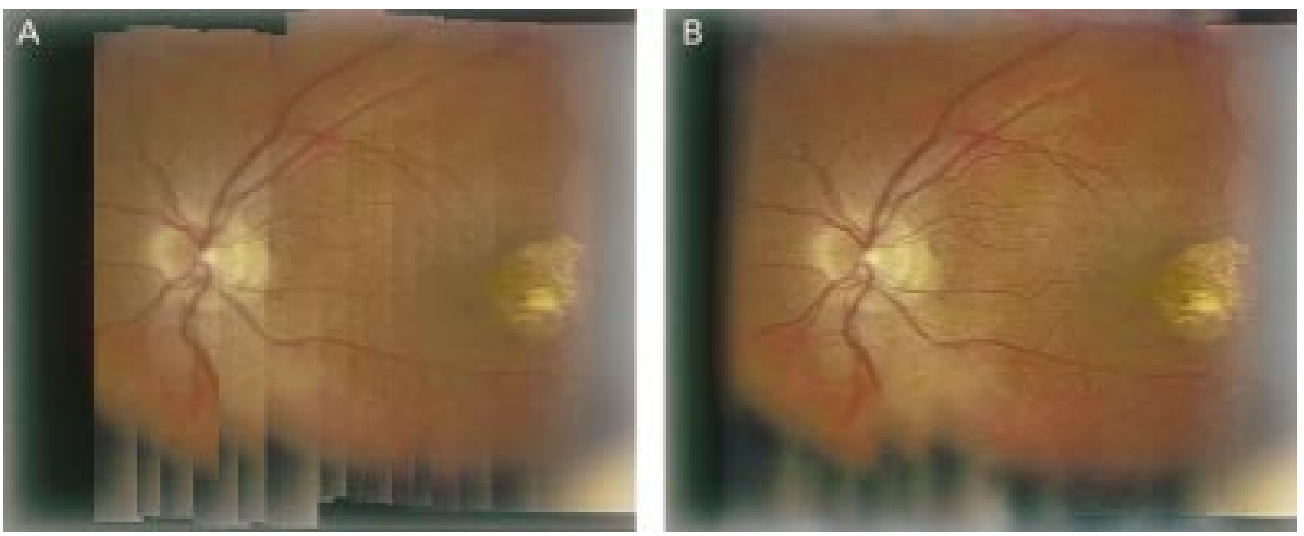

Figure 2 (A) Unblended mosaic of slit lamp fundus biomicroscope images for the eye depicted in Figure 1. (B) Final blended mosaic of slit lamp derived fundus biomicroscopic images.

green and red channels, but with a small blue channel component. ${ }^{1}$

Software was custom developed in $\mathrm{C}++$. A 35 second segment of video was selected, and digitised at $640 \times 480$ pixels. At 30 frames per second, an image sequence of 1050 individual frames was generated. Capitalising on image redundancy, and in order to limit computational requirements, every fifth frame was selected for analysis and processing. Pairwise image alignment was accomplished by minimising the sum of the squares of the differences (SSD) between the images with search over translation only. Following alignment and mosaicking of the first two images, subsequent images are aligned with, and added to, the intermediate mosaic by minimising the SSD metric. A final, unblended mosaic is generated.

The unblended mosaic contains prominent seams at the intersection between neighbouring fundus slit images. Blending may be accomplished with a number of filtering or signal averaging approaches. In this application, blending is accomplished following decomposition of each fundus slit image into a Laplacian pyramid representation. The final mosaic is formed by reconstructing the full resolution image from the Laplacian pyramid. ${ }^{2}$

\section{Results}

Alignment of multiple, partially overlapping slit lamp fundus images yields a high quality, wide field fundus image. An example of an eye with ocular histoplasmosis syndrome status post-juxtafoveal laser treatment for choroidal neovascularisation is depicted (Figs 1 and 2). The spatial relation between the optic nerve, fovea, and juxtafoveal scar cannot be appreciated by review of the individual slit lamp fundus biomicroscopic images (Fig 1), but is clearly depicted in the resultant mosaic (Fig 2).

Once the image set is mutually aligned (Fig 2A), blending algorithms reduce colour and luminance heterogeneity across the composite image. Seams, corresponding to abrupt changes in colour and brightness, are minimised or eliminated (Fig 2B).

\section{Discussion}

To our knowledge, these studies are the first investigations towards mosaicking slit lamp biomicroscope fundus image sequences.
Mosaicking algorithms have been developed for various applications with success, ${ }^{3}$ and commercial products are now available for mosaicking consumer camera imagery. In addition, alignment of the omnipresent, high contrast retinal vessels has permitted manual, computer assisted mosaic construction from fundus camera images, as well as progress towards semiautomated mosaicking of fundus camera and scanning laser ophthalmoscope (SLO) derived images. ${ }^{4}$

Fundus camera derived images, whether film based or digitally acquired, are high quality and suitable for grading and image analysis. ${ }^{6-8}$ In comparison with fundus camera or SLO images, slit lamp biomicroscopic fundus images are of a much reduced field of view and often of lower contrast. In addition, these images may be degraded by specular reflections arising from the examination lens, the cornea, and the sclera. Therefore, extraction of clinically useful images from this platform is challenging.

The slit lamp biomicroscope is ubiquitous in clinical practice, and is the workhorse for ophthalmic diagnosis and treatment; progress has been made towards the development and deployment of a slit lamp based platform for image guided diagnosis and treatment of retinal diseases. ${ }^{9-11}$ Mosaicking of slit lamp derived images is useful in that images for photodocumentation may be acquired during patient examination, obviating the need for specific fundus camera photographic sessions for eyes with selected disc and macular abnormalities. In addition, while all clinical ophthalmic and optometric practices have one or more slit lamp biomicroscopes, not all practices have access to a fundus camera. The method presented for slit lamp based photodocumentation requires a slit lamp, a computer, a slit lamp video adapter, and software. The first two of these are ubiquitous, while the slit lamp video adapter is becoming increasingly popular and less expensive.

In its raw form, the video image sequence requires approximately 27 megabytes per second $(640 \times 480 \times 3$ colour channels $=0.9$ megabytes per frame at 30 frames per second) for storage, while much of the data are extraneous or redundant. However, high quality, wide 
field of view, slit lamp based mosaic images can be transmitted to a remote location for communication, documentation, and consultation, and serve as an efficient distillation of the video image sequence for storage as might be appropriate for an electronic medical record. The images shown in Figure 2 occupy approximately 100 kilobytes of storage in high quality JPEG format.

In conclusion, we demonstrate that since images are typically acquired at video rates (30 frames per second), redundant data can be exploited to generate high quality mosaics of slit lamp biomicroscopic fundus images. Video image acquisition and image sequence processing algorithms allow for the construction seamless montages which efficiently distil the relevant image sequence data. The improved quality and wide field of view may confer suitability for inexpensive, real time photodocumentation of disc and macular abnormalities.

Supported by a career development award, Research to Prevent Supported by a career development award, Rese
Blindness (JWB), and NIH K-08 00374 (JWB).

Presented in part at the 2000 meeting of the Association for Research in Vision and Ophthalmology.
1 Madjarov BD, Berger JW. Automated, real-time extraction of fundus images from slit lamp fundus biomicroscope video image sequences. Br f Ophthalmol 2000;84:645-7.

2 Burt PJ, Adelson EH. The Laplacian pyramid as a compact image code. IEEE Transactions on Communications 1983;31: 532-40.

3 Sawhney HS, Hsu S, Kumar R. Robust video mosaicking through topology inference and local to global alignment. In: Proc ECCV 98. Freiburg, Germany. June 1998:Vol $2: 103-19$

4 Can A, Stewart CV, Roysam B. Robust hierarchical algorithm for constructing a mosaic from images of the
curved human retina. Proc IEEE Comp Soc. CVPR 1999 .

5 Rivero ME, Bartsch DU, Otto T, et al. Automated scanning laser ophthalmoscope image montages of retinal diseases. Ophthalmology 1999;106:2296-300.

6 Lee MS, Shin DS, Berger JW. Grading, image analysis and stereopsis of digitally compressed images. Retina 2000;20: stereopsis

7 Shin DS, Javornik NB, Berger JW. Computer-assisted, interactive fundus image processing for macular drusen quantitation. Ophthalmology 1999:1119-25.

8 Berger JW, Patel T, Shin DS, et al. Computerized stereochronoscopy and alternation flicker for optic nerve head contour change detection. Ophthalmology 2000;107: 1316-20.

9 Berger JW, Leventon M, Hata N, et al. Design considerations for a computer-vision-enabled ophthalmic augmented reality environment. Lecture Notes in Computer Science 1997;1205:399-408.

10 Berger JW, Shin DS. Image-guided macular laser therapy: design considerations and progress towards implementation. Proc SPIE (Ophthalmic Technologies) 1999;3591:2417 .

11 Berger JW, Shin DS. Computer-vision-enabled augmented reality fundus biomicroscopy. Ophthalmology 1999;106: 1935-41.

\section{Contributors please note:}

Communications from all countries except the UK and Republic of Ireland should be sent to Professor C Hoyt, Editor, British fournal of Ophthalmology, University of California, Department of Ophthalmology, 10 Kirkham Street, K 301, San Francisco, CA 94143-0730, USA (tel: 001415 502-6871; fax: 001415 514-1521).

Manuscripts from the UK and the Republic of Ireland should be sent to Professor Andrew Dick, UK Editor, British fournal of Ophthalmology, Division of Ophthalmology, University of Bristol, Lower Maudlin Street, Bristol BS1 2LX (tel: +44 (0) 0117 929-4496; fax: +44 (0) 117 929-4607). 